\title{
Interventions for Autism Spectrum Disorders
}

Stephen E. Brock

Kim Silva

Annie Riffey

Silvia Ludena

California State University Sacramento

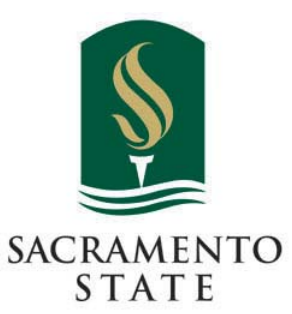

\author{
$58^{\text {th }}$ Annual \\ 2007 CASP Convention \\ Los Angeles, CA \\ March 9, 2007
}




\section{Sample Psycho-educational Report Recommendations}

From a review of the literature regarding the school psychologist's role in identifying, assessing, and treating autism spectrum disorders (ASD; Brock, Jimerson, \& Hansen, 2006), we have identified interventions often recommended when addressing some of the specific challenges associated with these disorders. This presentation, and the following handout, offers some of these recommendations (along with the accompanying background information) that we feel you might find useful when writing a psycho-educational report. It is important to acknowledge that without a careful assessment of specific student needs this document will not be helpful. However, following a comprehensive psycho-educational evaluation, and the identification of specific student needs, we feel that this information will be helpful in stimulating your thinking about appropriate psycho-educational report recommendations for the student with ASD. 


\section{Social Relations}

\section{If the student is challenged by social situations, then the following intervention and support recommendations might be appropriate:}

1. Provide interpretation of social situations. Specifically, the following are suggested:

a. Make use of social stories ${ }^{\mathrm{TM}}$ (Gray \& White, 2002). A social story is a short story that explains a specific challenging social situation. The goal is to find out what is happening in a situation. The following is an example of a social story:

When other students Get upset
Sometimes other students get upset and cry.
When this happens their teacher might try to help them.
The teacher might try to help them by talking to them or holding them.
This is okay.
Sometimes when other students get upset and cry, it makes me upset and angry.
I can use words to tell my teacher that I am upset.
I can say, "That makes me mad!" or "I'm upset!"
It is okay to use words about how I feel.
When I get upset I will try to use words about how I feel.

i. For more information about social stories go to

- http://www.thegraycenter.org/

- http://www.polyxo.com/socialstories/introduction.html

ii. A variety of sample stories can be found at

- http://www.frsd.k12.nj.us/autistic/Social\%20Stories/social_stories.htm

b. Use cartooning to illustrate the rules of challenging social situations (Myles \& Simpson, 2001). For example, ...

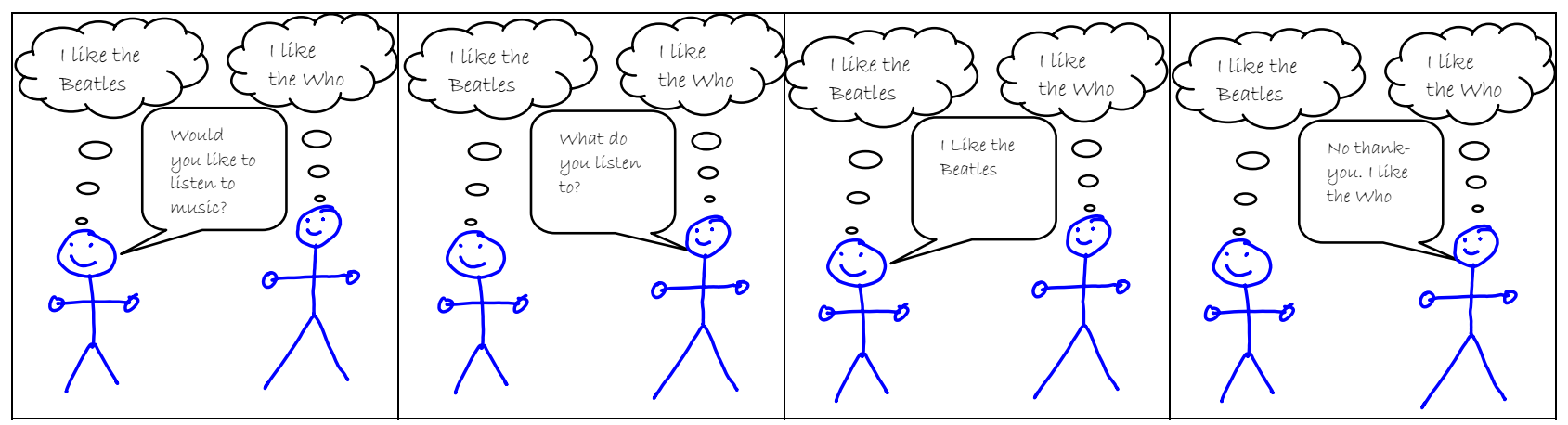

c. Explain problematic social situations and in doing so let NAME know that there are specific choices to be made and that each choice has a specific consequence. Specific steps in this process (as described by Myles \& Simpson, 2001) are as follows:

i. Help NAME understand the problematic social situation (i.e., who was involved, what happened, etc.).

ii. Facilitate NAME's brainstorming of options for responding to the situation.

iii. Help NAME explore the consequences for each option identified.

iv. Help NAME identify the response that has the most desirable consequences.

v. Develop and action plan. 
vi. Practice the response to the problematic social situation by role playing, visualizing, writing a plan, or talking it out with a peer.

d. To address NAME's difficulty making friends, the following interventions are recommended:

i. Establish structured activities with peers. These activities should have preassigned roles that can be practiced.

ii. Provide direct instruction on how to approach an individual or group.

iii. Provide direct instruction on the skills needed to interact with peers.

iv. Structure social opportunities around NAME's special interests.

e. After a challenging social situation conduct a "social autopsy" (Myles \& Simpson, 2001). Such a conversation involves an examination and inspection of NAME's social errors to discover their causes, better understand the consequences of such errors, and to decide what can be done to prevent it from happening again.

f. Identify specific social conventions that need to be taught and then provide direct instruction. Examples, of social conventions that NAME may need to be taught include the following (LIST HERE SPECIFIC SOCIAL RULES THAT THE ASSESSMENT DATA SUGGESTS MAY NEED TO BE TAUGHT. EXAMPLES INCLUDE THE FOLOWING):

i. "Do not ask to be invited to someone's party

ii. Speak to teachers in a pleasant tone of voice because they will respond to you in a more positive manner. They also like it if you smile every once in a while.

iii. Do not correct someone's grammar when he or she is angry.

iv. Never break laws - no matter what your reason.

v. When your teacher gives you a warning about your behavior and you continue the behavior, realize that you probably are going to get in trouble. If you stop the behavior immediately after the first warning, you will probably not get in trouble.

vi. Do not touch someone's hair even if you think it is pretty.

vii. Do not ask friends to do things that will get them in trouble.

viii. Understand that different teachers may have different rules for their classes.

ix. Do not draw violent scenes.

$\mathrm{x}$. Do not sit in a chair that someone else is sitting in - even if it is 'your' chair.

xi. Do not argue with a policeman - even if you are right.

xii. Do not tell someone you want to get to know better that he or she has bad breath.

xiii. Do not try to do what actors do on television or the movies. These shows are not the same as real life.

xiv. Do not pick flowers from someone's garden without permission, even if they are beautiful and you want to give them to someone” (Myles \& Simpson, 2001, p. 8). 
g. Make use of NAME's special interests to develop "power cards" that facilitate the understanding of social rules (Myles \& Simpson, 2001). (TRY TO LINK THE STUDENTS SPECIAL INTERESTS TO PROBLEMATIC SOCIAL SITUATIONS.) For example, make use of NAME's interest in automotive mechanics and provide him/her with the following card that can be placed on his/her desk and/or in his/her pocket.

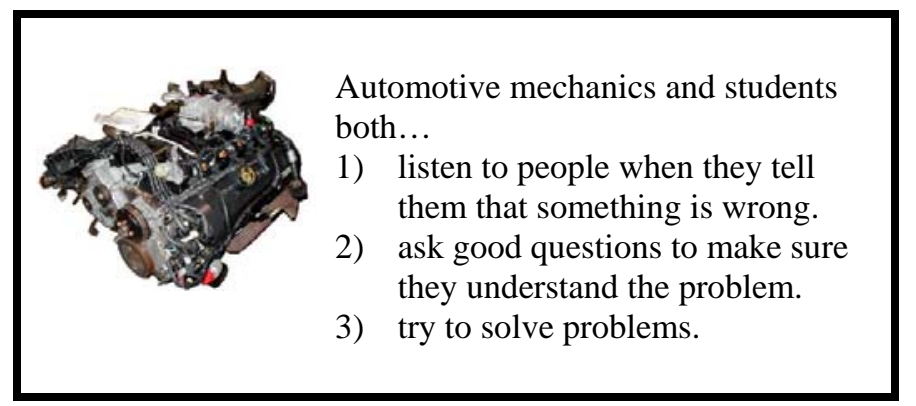

\section{Communication}

If the student has difficulties with expressive language, then the following might be appropriate:

1. Consider making use of a Picture Exchange Communication System (PECS; Frost \& Bondy, 1994; Preis, 2006). PECS is a picture based communication system wherein the student gives a picture or symbol of a desired item in exchange for the item itself. The intent of PECS is to assist the student in developing spontaneous communication. The following are examples of PECS symbols:

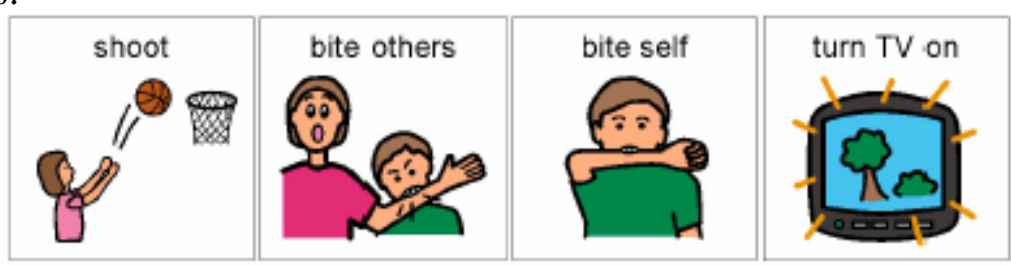

a. Sample PECS IEP objectives can be found at http://www.pecs.com/Brochures/Objectives/IEP\%200bjectives\%202002.pdf

b. PECS pictures and photos can be found at http://www.childrenwithspecialneeds.com/downloads/pecs.html

c. Blank PECS image grids, and daily and weekly picture card schedule forms can be found at http://www.do2learn.com/picturecards/forms/index.htm

d. For more information about PECS go to

i. http://www.bbbautism.com/pecs_contents.htm

ii. http://www.polyxo.com/visualsupport/pecs.html

iii. http://www.usd.edu/cd/autism/topicpages/printer/PECS.pdf

iv. http://www.nas.org.uk/nas/jsp/polopoly.jsp?d=297\&a=3642\&view=print

v. http://www.iidc.indiana.edu/irca/communication/WhatisthePEC.html 
e. Specific PECS cards should include the following (AS INDICATED BY ASSESSMENT DATA):

i. "Break" Cards that assist NAME in communicating when he/she needs to escape a task or situation.

ii. "Choice" cards that provide NAME some control by indicating a choice from a prearranged set of possibilities.

iii. "All done” cards that assist NAME in communicating when he/she is finished with an activity or task.

iv. "Turn-taking” cards that can be used to visually represent and mark whose turn it is.

v. "Wait" cards that can be used to visually teach the concept of waiting.

vi. "Help" cards that assist in teaching NAME to raise his/her hand to indicate the need for assistance.

If the student has difficulties with receptive language, then the following might be appropriate:

1. Consider using a Picture Exchange Communication System (Preis, 2006) paired with a verbal command.

a. When giving a verbal command, hand NAME the corresponding PECS icon at the same time the verbal command is given. This will provide NAME with both a verbal and visual cue to assist him/her in understanding what is expected of him/her.

b. Providing paired verbal and visual cues with PECS will increase NAME's generalization of language concepts across environments (i.e., people and settings).

If the student has difficulties with receptive and expressive vocabulary, then the following might be appropriate:

1. Consider using total communication to teach and speak to NAME (Goldstein, 2002). The following strategy is recommended:

a. When speaking to NAME, pair your speech with the appropriate corresponding sign. For example, ...

Say: “Cookie” and make the sign below

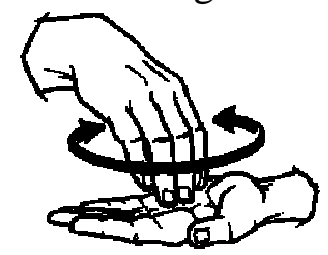

* To make the sign: The right hand, in the ' $\mathrm{C}$ ' ?position, palm down, is placed into the open left palm. It then rises a bit, swings or twists around a little, and in this new position is placed again in the open left palm. 
b. For more information about how to use sign language go to http://commtechlab.msu.edu/sites/aslweb/browser.htm

1. Consider using the Prelinguistic Milieu Training (PMT) with NAME (Goldstein, 2002). Specific PMT strategies (as described by McCathren, 2000) include the following:

a. Following NAME's attentional leads (i.e., talking about and playing with NAME's focus of interest).

b. Using prompts for communication (i.e., asking for specific behaviors).

c. Using behavioral and vocal imitation (e.g., NAME claps and vocalizes, adult claps and vocalizes).

d. Modeling conventional gestures (i.e., pointing, nodding) and vocalizations with consonants.

e. Developing play routines (i.e., rolling a toy truck back and forth, etc.).

f. Changing the environmental arrangement.

g. For more information on Prelinguistic Milieu Training (PMT) go to http://childconnections.tripod.com/id13.html.

If the student has difficulties initiating conversation with others, then the following might be appropriate:

1. Consider pairing NAME with a peer tutor during social play time (Whitaker, 2004). The following strategies are recommended:

a. Provide NAME with a structured play time during which highly desirable toys are provided.

b. Have the peer tutor participate with NAME during the structured play time.

c. Have the peer tutor get close to NAME during the play time, follow NAME's lead during the play time, talk slowly and simply to NAME, and make the play time fun.

d. Have the peer tutor join in any activity that NAME spontaneously chooses.

If the student has difficulties initiating or sustaining conversation with others, then the following might be appropriate:

1. Consider using a cue card/written script program to develop conversational skills. Specific steps in this program (as described by Charlop-Christy \& Kelso, 2003) are as follows:

a. Sit across from NAME and ask him/her the initial conversation question for a predetermined conversation. For example, ...

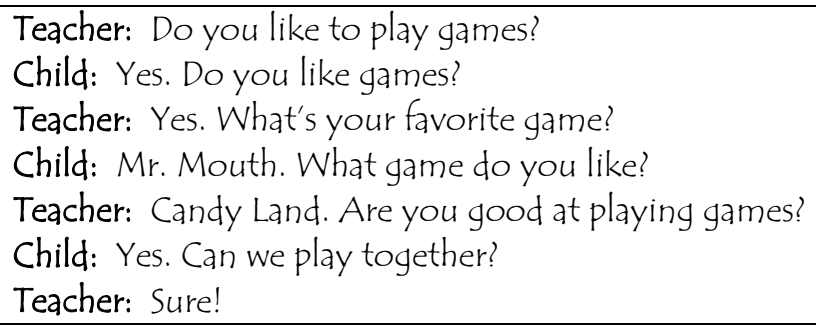

b. Immediately present NAME with a cue card upon which a scripted response and question are written and ask NAME to "read it out loud." 
c. Next, ask NAME to repeat the scripted line to you (e.g., teacher, classroom assistant, etc.) while maintaining eye contact.

d. Repeat the above procedure for each of NAME's lines in the conversation.

e. Upon correct completion of the conversation, give NAME reinforcement (e.g., praise, a high five, etc.) for good reading, sitting, and attention.

f. Next, remove all cue cards and ask NAME the initial conversation question again.

\section{Challenging Behaviors}

If disruptive behavior problems are present, then the following might be appropriate:

1. Functional behavioral assessment is recommended.

a. Students with autism frequently engage in disruptive behaviors to escape demands and gain or maintain access to perseverative items and activities (Reese, Richnam, Zarcone, \& Zarcone, 2003). Thus, the focus of any functional assessment should include special attention to perseverative behaviors that might serve to obtain desirable sensory stimuli.

b. Students with autism also frequently engage in disruptive behaviors to escape aversive sensory stimuli (Reese et al., 2003). Thus, the focus of any functional assessment should also direct attention to perseverative behaviors that might serve to escape from aversive sensory stimuli.

c. From the functional behavioral assessment, determine if differential reinforcement of alternative, other, or incompatible behavior will be necessary. The technique employed should derive from the behavioral assessment (Dozier, Iwata, \& Neidert, 2005).

If a student needs predictability (e.g., becomes anxious when new materials/activities are introduced), then the following might be appropriate:

1. Employ “priming” (Myles \& Adreon, 2001). This involves showing the actual instructional materials that will be used in a lesson the day, evening, or morning before the given classroom activity is going to take place. Priming should be brief (10 to 15 minutes) and built into NAME’s daily schedule and should take place in a relaxing environment.

2. Employ a visual schedule of new tasks (Dettmer, Simpson, Myles, \& Ganz, 2000). This involves showing the student a pictographic sequence of the events that will unfold. The schedule should give NAME information of the activity and when it will be finished.

3. Employ the use of social stories (Edwards, Rabian, Scattone, \& Wilczynski, 2002). This involves informing NAME of the new events that will be experienced and appropriate ways to act in these situations.

If disruptive behavior problems are present and known to be related to perseverative activities, then the following might be appropriate (Reese et al., 2003):

1. Identify and decrease environmental and/or physiological conditions that are related to perseverative behavior. 
2. Determine if the behavior is an attempt to avoid aversive sensory stimulation or a strategy to obtain desirable sensory stimulation.

If disruptive behaviors appear to be related to anxiety and/or a desire to avoid aversive sensory stimulation, then the following might be appropriate (Reese et al., 2003):

1. The problem (perseverative) behaviors appear to have a calming or organizing effect and might be related to anxiety. Thus, the following strategies are recommended as they appear to reduce anxiety (and in doing so may decrease the need for the perseverative behaviors):

a. Establish predictable routines

b. Use visual schedules to facilitate coping with change

c. Practice alternative coping behaviors such as relaxation

If disruptive behaviors appear to be related to obtaining desirable sensory stimulation, then the following might be appropriate (Reese et al., 2003):

1. The problem (perseverative) behavior(s) appear to be positively reinforcing. Thus, the following strategies are recommended:

a. Provide appropriate access to the desired sensory stimulation on a regular basis. Provide instruction on how to appropriately obtain the desired stimuli. This will decrease the need to engage in behaviors that have as their function obtaining the stimuli.

b. Providing contingent access to the desired sensory stimulation may be used as a positive reinforcer for the completion of instructional tasks.

Further recommendations for students whose sensory issues are judged to play a role in specific problem behaviors include the following (adapted from Myles, Cook, Miller, Rinner, \& Robbins, 2000):

1. To address NAME's problems making eye contact, the following interventions are recommended:

a. Consider decreasing expectations for eye contact in some situations.

b. Try to place speakers in NAME's line of sight with out getting too close

c. Strive to provide minimal auditory information and/or offer slight touch to encourage visual attention

2. To address NAME's difficulties understanding body language and/or facial expressions the, following interventions are recommended:

a. Provide auditory cues to direct NAME's attention.

b. Try to eliminate irrelevant background distractions.

c. Strive to pair facial expressions, gestures, and body language with words.

d. Strive to be cognizant of unspoken social cues when giving instructions.

3. To address NAME's difficulty transitioning in hallways, the following interventions are recommended:

a. Allow NAME to be either first or last in line.

b. Allow NAME to leave class early.

c. Have NAME carry something heavy to provide proprioceptive input. 
4. To address NAME's constant humming, the following interventions are recommended:

a. Move NAME away from noise sources that may be distressing

b. Allow NAME to hum, but teach him/her that such is appropriate only in certain situations (e.g., to help him/her concentrate) and try to get him/her to do it more quietly.

5. To address NAME's desire to touch, the following interventions are recommended:

a. Before NAME enters a new environment specify exactly what can/cannot be touched.

b. Before NAME enters a new environment provide deep pressure by rubbing his/her shoulder, back, or palms.

6. To address NAME's messy handwriting, the following interventions are recommended:

a. Have NAME engage in gross-motor activities before being asked to perform finemotor tasks.

b. Encourage NAME to engage in activities that develop hand strength.

c. Have NAME write on raised-line paper

d. Teach NAME keyboarding skills.

\section{Academic Functioning}

If the student has weaknesses in, attention, organizational, transitional, and auditory processing, then the following might be appropriate:

1. To address NAME's poor organizational skills, the following interventions are recommended (adapted from Myles, Cook, Miller, Rinner, \& Robbins, 2000):

a. Provide as much visual structure as is possible

b. Use tape and labels to specify where instructional materials are to be placed.

2. To address NAME's difficulty with change, the following interventions are recommended(adapted from Myles, Cook, Miller, Rinner, \& Robbins, 2000):

a. Offer a signal before transitions take place

b. Use visual cues to prepare NAME for what will happen next

c. Allow NAME to obtain deep pressure

d. Give NAME a script or social story to follow whenever an unexpected event takes place.

3. The instructional program should center on NAME's strengths (TYPICALLY ROTE MEMORY AND VISUAL PROCESSING), special interests, and needs (Dettmer et al., 2000; Quill, 1997). It may include the following:

a. Visual schedules that depict the student's daily routine

b. Work systems

c. Calendars to help the student understand when regularly scheduled events may occur

d. To facilitate transitions, make use of visual cues that forewarn the student when something is going to end, stop or be all done. This assists in transitions. This can be done with a visual count down timer.

e. Place classroom rules in a visual form on the student's desk.

f. Place strong visual cues throughout the classroom to guide the student through physical space.

i. Use boundary markers such as barriers, rugs, bookcases, other furniture, or colored tape on the floor to represent boundaries of areas for play and study. 
ii. Use movable signs to mark spaces that are used for a particular purpose at a specific time.

iii. Store common classroom materials (e.g., school supplies, games) on accessible shelves or in see through storage containers. When needed, provide labels for these materials (using pictures paired with words).

g. Use visual cues in instruction (e.g., hands-on demonstrations and modeling, objects, pictures) as needed to help NAME better grasp the directions.

h. Use strategies to make directions and learning expectations clearly understood.

i. Include essential and concrete information in directions that will answer: (1) How much work is there to do in this task? (2) What exactly am I supposed to do? (3) When do I do the work? (4) What is my payoff for doing the work? (Volmer, 1995).

If a student has reading fluency and/or comprehension difficulties, then the following might be appropriate:

1. Highlighted text

2. Study guides

3. Graphic Organizers (Pictorial representations may be substituted for words; Ae-Hwa Kim, 2004).

\section{Graphic Organizer Examples}

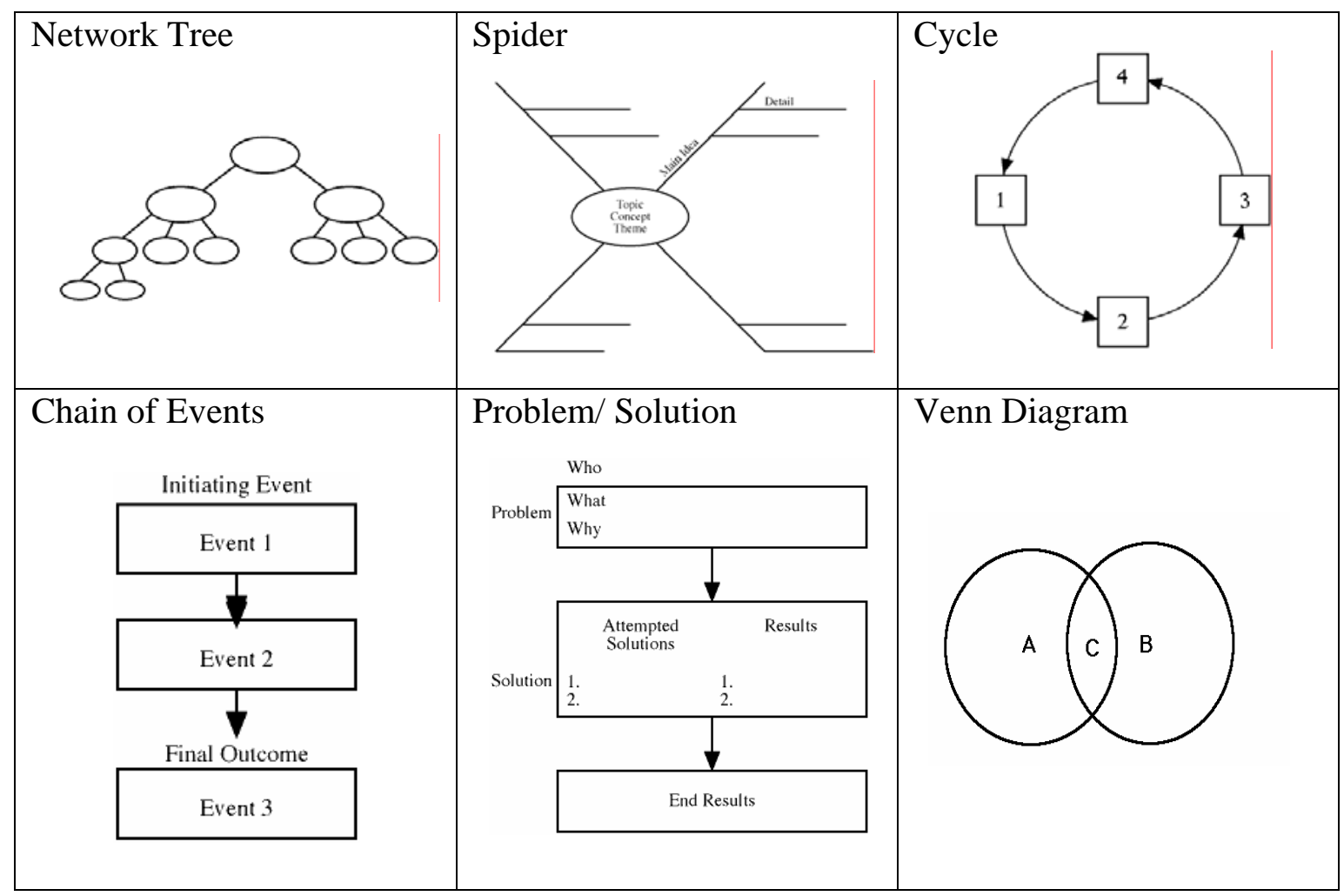


If a student has written expression (e.g., handwriting) difficulties, then the following might be appropriate:

1. When assessing NAME's content knowledge allow for verbal, instead of written responses.

2. When completing written assignments allow NAME to use the computer instead of pen or pencil.

3. Multiple-choice tests can be used instead of short answer to assess subject matter knowledge.

4. Allow NAME to create projects, rather than producing written reports.

If a student has difficulty with note taking, then the following might be appropriate:

1. Provide NAME with a complete outline including the main idea and supporting details of a lesson or lecture.

2. Provide NAME with a skeletal outline that he/she can use to fill in details.

3. Provide NAME with a peer copy of the notes

\section{Alternative Treatments}

If parents are interested in other treatments to help reduce symptoms or behaviors associated with autism, then the following is a list of recommendations that may be a part of a comprehensive treatment plan. However, the school psychologist should treat these recommendations with caution, as there is limited research to support their effectiveness, and they would NOT typically appear in the psycho-educational report.

If the student exhibits behavioral problems, then the following vitamin supplements might be recommended (Levy \& Hyman, 2002):

- Combination of vitamin B6 and magnesium

- Vitamin C

- Vitamin A

If the student exhibits behavioral problems, then the following medications might be recommended (Levy \& Hyman, 2002; http://www.fda.gov/bbs/topics/NEWS/2006/NEW01485.html):

- Secretin

- Risperdal

For regulation of immune function, the following treatments might be recommended (Levy \& Hyman, 2002):

- Antibiotic

- Antifungal

- Antiviral Medications

- Probiotics

- Intravenous human immune globulin 
- Vitamin A supplementation

- Withholding of vaccines

For control or improvement of symptoms of autism, the following might be recommended (Levy \& Hyman, 2002):

- Gluten-free/casein-diet

- Chelatin/Mercury-detoxification

If the student is sensitive to sound, then the following might be appropriate (Levy \& Hyman, 2002):

- Use of auditory integration to decrease the sensitivity to sound through systematic exposure to altered music by headphones.

If the student experiences communication challenges, then the following might be recommended (Levy \& Hyman, 2002):

- Use of facilitated communication.

If the student exhibits behavior problems, then the following might be recommended (Levy \& Hyman, 2002):

- Use of craniosacral manipulation. 


\section{References}

Ae-Hwa Kim, B. A., Vaughn, S., Wanzek, J., \& Shangjin, J. (2004). Graphic organizers and their effects on the reading comprehension of students with LD: A synthesis of research. Journal of Learning Disabilities, 37, 105-118.

Brock, S. E., Jimerson, S. R., \& Hansen, R. L. (2006). Identifying, assessing, and treating autism at school. New York: Springer.

Charlop-Christy, M., \& Kelso, S. (2003). Teaching children with autism conversational speech using a cue card/written script program. Education and Treatment of Children, 26, 108-119.

Dettmer, S., Simpson, R. L., Myles, B. S., \& Ganz, J. B. (2000). The use of visual supports to facilitate transitions of students with autism. Focus on Autism and Other Developmental Disabilities, 15, 163-169.

Dozier, C.L., Iwata, B. A., \& Neidert, P. L. (2005). Treatment of multiply controlled problem behavior with procedural variations of differential reinforcement. Exceptionality, 13(1), 45-53.

Edwards, R. P., Rabian, B., Scattone, D., \& Wilczynski, S. M. (2002). Decreasing disruptive behaviors of children with autism using social stories. Journal of Autism and Developmental Disorders, 32, 535-543.

Frost, L., \& Bondy, A. (1994). The picture exchange communication system training manual. Cherry Hill, NJ: PECS.

Goldstein, H. (2002). Communication intervention for children with autism: A review of treatment efficacy. Journal of Autism and Developmental Disorders, 32, 373-396.

Gray, C., \& White, A. L. (2002). My social stories book. Gateshead, Great Britain: Arthenaeum Press.

Levy, S., \& Hyman, S. (2002). Alternative/complementary approaches to treatment of children with autistic spectrum disorders. Infants and Young Children, 14, 33-42.

McCathren, R. (2000). Teacher implemented prelinguistic communication intervention. Focus on Autism and Other Developmental Disabilities, 15, 21-29.

Myles, B. S., \& Adreon, D. (2001). Asperger syndrome and adolescence: Practical solutions for school success. Shawnee Mission, KS: AAPC.

Myles, B. S., Cook, K. T., Miller, N. E., Rinner, L., \& Robbins, L. A. (2000). Asperger syndrome and sensory issues: Practical solutions for making sense of the world. Shawnee Mission, KS: AAPC.

Myles, B. S., \& Simpson, R. L. (2001, November). Effective practices for students with Asperger syndrome. Focus on Exceptional Children, 34(3), 1-14.

Preis, J. (2006). The effect of picture communication symbols on the verbal comprehension of commands by young children with autism. Focus on Autism and Other Developmental Disabilities, 21, 194210.

Quill, K. A. (1997). Instructional considerations for young children with autism: The rational for visually cued instruction. Journal of Autism and Developmental Disorders, 27, 697-714.

Reese, R. M., Richnam, D. M., Zarcone, J., \& Zarcone, T. (2003). Individualizing functional assessments for children with autism: The contribution of perseverative behavior and sensory disturbances to disruptive behavior. Focus on Autism and Other Developmental Disabilities, 18, 87-92.

Volmer, L. (1995). Best practices in working with students with autism. In A. Thomas \& J. Grimes (Eds.), Best practices in school psychology (3rd ed., pp. 1031-1038). Bethesda, MD: National Association of School Psychologists.

Whitaker, P. (2004). Fostering communication and shared play between mainstream peers and children with autism: Approaches, outcomes, and experiences. British Journal of Special Education, 31, 215-222. 